\title{
Efficacy and safety of Acupuncture for Gastrointestinal Dysfunction after Laparoscopy: study protocol for a randomized controlled trial
}

LISHA LIU ( $\square$ wenliyinyusha@163.com )

Mianyang hospital affilicated to Chengdu university of TCM https://orcid.org/0000-0001-8965-4366

\section{XIULI YUAN}

Mianyang Hospital affiliated to Chengdu university of TCM

QUANMIN JIANG

Chengdu University of Traditional Chinese Medicine

\section{LEI YANG}

Mianyang affiliated hospital of Chengdu university of TCM

\section{GUANGQIANG HUANG}

Mianyang Anzhou hospital

\section{JINGYUAN ZHANG}

Chengdu University of Traditional Chinese Medicine

\section{LUO JING}

Mianyang third hospital

\section{XIAOYING LIU}

MianYang Anzhou people's hospital

JIAN HUO

Chengdu University of Traditional Chinese Medicine

\section{Study protocol}

Keywords: acupuncture, gastrointestinal dysfunction, postoperative

Posted Date: December 19th, 2019

DOl: https://doi.org/10.21203/rs.2.19289/v1

License: (c) (i) This work is licensed under a Creative Commons Attribution 4.0 International License.

Read Full License 


\title{
Efficacy and safety of Acupuncture for Gastrointestinal
}

\section{Dysfunction after Laparoscopy: study protocol for a randomized}

\section{controlled trial}

\author{
Lisha Liu ${ }^{1}$, Xiuli Yuan ${ }^{1}$, QuanminJiang ${ }^{2}$, Lei Yang ${ }^{1}$, Guangqiang Huang ${ }^{4}$, Jingyuan Zhang ${ }^{2}$, \\ Jing Luo ${ }^{3}$, Xiaoying Liu ${ }^{4}$, and Jian $\mathrm{Huo}^{2}$
}

(1. Mianyang Hospital affiliated to Chengdu University of Traditional Chinese Medicine, 2. ChengDu University of Traditional Chinese Medicine, 3. Mianyang Third Hospital,4. Mianyang Anzhou Hospital, 621000, Mianyang, China)

\section{Abstract \\ Background}

Postoperative gastrointestinal dysfunction is one of the common complications after surgery. At present, there has no internationally recognized acupuncture program although it has a positive effect on the recovery of postoperative gastrointestinal function. In this report, we have designed a scientific trial protocol to analyze the feasibility of applying acupuncture to gastrointestinal dysfunction after laparoscopic surgery. We intend to conduct a randomized controlled trial based on this protocol to evaluate the efficacy and safety of the acupuncture for postoperative gastrointestinal dysfunction.

\section{Method / design}

This study is a randomized, evaluator blinded, controlled, multi-center clinical trial, which has been designed according to the Consolidated Standards of Reporting Trials (CONSORT 2010) and the standard for Reporting Interventions in Controlled Trials of Acupuncture (STRICTA). The subjects will be chosen from the inpatients who have undergone laparoscopic surgery of Mianyang Hospital Affiliated to Chengdu University of traditional Chinese medicine, Mianyang Third People's Hospital and Mianyang Anzhou District People's Hospital. According to the random number generated by SPSS 25.0 software, the qualified subjects will be randomly assigned to the experimental group, the control group, and the blank group. Therapies will be performed 30 minutes after operation, the experimental group will be treated with acupuncture, the control group will be treated with intravenous injection of granisetron, and the blank group will be treated with intravenous injection of physiological saline. The main outcome is the first time of flatus after operation, and the secondary outcomes are abdominal pain, abdominal distention, nausea, vomiting times, gastrointestinal hormone level, and mental state. We will also evaluate the efficacy and safety of acupuncture on the basis of the principle of good clinical practice (GCP).

\section{Discuss}

This is a standardized and scientific clinical trial to evaluate the efficacy and safety of acupuncture for gastrointestinal dysfunction after laparoscopic surgery. Our goal is to provide objective evidence and improve the acupoint prescription in clinical practice, so as to promote the clinical application of this technology.

\section{Trial registration}


China clinical trial registration ID: CHICTR -1800016991. Registered on 6 July 2018.

\section{Background}

Postoperative gastrointestinal dysfunction is one of the common complications after surgery, which can lead to nausea, vomiting, abdominal pain, bloating, constipation, diarrhea, and other clinical symptoms ${ }^{[1]}$. Postoperative gastrointestinal dysfunction may even cause intestinal paralysis, acute gastric dilatation, and obstructions in severe cases, which affect patients' quality of life and prolong the hospital stay of the patients ${ }^{[2]}$. At present, the specific mechanism of postoperative gastrointestinal dysfunction is not totally clear. It is known that postoperative gastrointestinal dysfunction is closely related to general anesthesia, electrolyte disturbance, abdominal inflammation, and gastrointestinal hormones ${ }^{[3]}$. The routine treatment of gastrointestinal function recovery includes fasting, enema, oxygen inhalation, analgesia, fluid supplement, acid-base regulation, infection prevention, and gastrointestinal decompression ${ }^{[4]}$. Granisetron, domperidone, cisapride, and everypan are the representative drugs for postoperative gastrointestinal dysfunction. Domperidone and cisapride may cause adverse reactions of cardiovascular system, fever and headache may occur if granisetron is used, and side effects of nausea and vomiting may be caused by evermopan ${ }^{[5]}$. The side effects and risks of these drugs limit their wide application. Enhanced recovery after surgery(ERAS) ${ }^{[6]}$ refers to the application of all kinds of evidence-based therapies in perioperative period to reduce surgical stress and complications, accelerate postoperative recovery and shorten hospital stay of the patients. Although the ERAS has been paid more and more attention by surgeons, there is still a lack of active and effective intervention measures.

Acupuncture is a traditional alternative therapy which treat diseases by using the needle to stimulate the acupoints. A large number of experimental studies and clinical observations has shown that acupuncture has obvious two-way regulatory effect on the movement, secretion and absorption of the digestive system ${ }^{[7-9]}$. The regulating effect of acupuncture for gastrointestinal function may be achieved by stimulating nerves, especially vagus nerve. $\mathrm{Lu}^{[10]}$ Found that electroacupuncture at ST36 regulated gastric motility in rats through vagus and sympathetic reflex respectively mediated by $m 2 / 3$ and $\beta 1 / 2$ receptors, and vagus reflex played a major role in this procedure. The results of a research showed that ST36 could stimulate the peroneal nerve, and then transmit the pulse signal to the vagus nerve through the sciatic nerve, release the 1-amino acid decarboxylase and increase the neurotransmitter level ${ }^{[11]}$. Electroacupuncture at Neiguan (PC6) can stimulate the sensory fibers of the median nerve, and then stimulate the solitary tract nucleus of the vagus nerve, leading to the release of neurotransmitters ${ }^{[12]}$. With the development of the research on gastrointestinal diseases, more and more scientists believe there is a close relationship between gut and brain, and the brain-gut axis is the bridge between central nervous system and gastrointestinal tract $^{[13]}$. At present, the recognized brain-gut axis includes central nervous system, autonomic nervous system (ANS), hypothalamic pituitary axis (HPA) and brain-gut peptide ${ }^{[14]}$. Signals from the brain can affect gastrointestinal motility, whereas information from the Gastrointestinal tract can also affect brain function. Brain-gut peptide is a kind of small molecular peptide with double distribution in gastrointestinal tract and brain, which play an important role in gastrointestinal motility ${ }^{[15]}$. It has been shown that peripheral ghrelinis is critical in regulating gastrointestinal function, while ghrelin in central nervous system can affect gastrointestinal activity ${ }^{[16]}$. There is an experiment ${ }^{[17]}$ also showed that electroacupuncture can reduce auxin and neuropeptide $\mathrm{Y}$ in rats and reduce their food intake. Excessive release of 5-HT and SP from gastrointestinal tract may cause 
intestinal dysfunction, while scalp acupuncture can reduce the expression level of 5-HT and SP in irritable patients to promote the recovery of gastrointestinal function ${ }^{[18]}$.

In recent years, acupuncture is considered as one measure of ERAS by some researchers and has gradually been applied to postoperative complications. In 1998, the National Institutes of Health $(\mathrm{NIH})$ issued a consensus statement ${ }^{[19]}$ that acupuncture can be an effective treatment for postoperative nausea and vomiting. Some systematic reviews ${ }^{[20-22]}$ have investigated the effectiveness of acupuncture for postoperative gastrointestinal dysfunction and come to a positive conclusion. Due to the defects of research quality, statistics and research methods, the clinical results should be interpreted carefully and their scientific basis is still limited. Scalp acupuncture is a kind of therapy to treat diseases by stimulating specific parts of the head area, which has a good effect on many diseases caused by the brain ${ }^{[23]}$. The acupuncture prescriptions for postoperative gastrointestinal dysfunction mostly involve the points located in trunk and limbs ${ }^{[24]}$. While this clinical trial chose the acupoints in the head area on the basis of brain-gut axis theory, which is a new exploration and attempt to apply acupuncture for postoperative gastrointestinal dysfunction. 18 patients who undergone laparoscopic surgery had been pre tested strictly followed the evaluation and treatment process of this study. These subjects were randomly assigned to "experimental group" (acupuncture) and "control group" (granisetron), we observed that acupuncture can effectively treat gastrointestinal dysfunction after laparoscopic surgery in the pre experiment. Therefore, we designed this randomized controlled study to evaluate the effectiveness and safety of acupuncture compared with commonly used drug, and to determine the feasibility of acupuncture research in the future comprehensive randomized controlled trial (RCT).

\section{Methods and design}

\section{Study Design}

This study is a randomized, evaluator blinded, controlled, multi-center clinical trial that will be conducted in Mianyang Hospital Affiliated to Chengdu University of traditional Chinese medicine, Mianyang Third Hospital, Mianyang Anzhou District Hospital, China. Eligible participants will be randomly assigned to the experimental group $(n=90)$, the control group $(n=90)$, and the blank group $(n=90)$ with a ratio of 1:1:1. The patients will receive one of the following interventions 30 minutes after laparoscopic surgery: 1. Acupuncture, the needle will stay 30 minutes in body and 6 hours in head. 2. intravenous injection of granisetron; 3. intravenous injection of physiological saline. The general conditions and observation indexes of the subjects will be evaluated in baseline before operation, $2 \mathrm{~h}, 6 \mathrm{~h}, 12 \mathrm{~h}, 24 \mathrm{~h}, 48 \mathrm{~h}, 2$ weeks after discharge. The results will follow the Consolidated Standards of Reporting Trials (CONSORT) ${ }^{[25]}$ guidelines as well as Standards for Reporting Interventions in Clinical Trials for Acupuncture (STRICTA) ${ }^{[26]}$ and Standard Protocol Items: Recommendations for Interventional Trials (SPIRIT) guidelines ${ }^{[27]}$. This study (protocol version 2.0, 25 May 2018) has been registered at the Chinese Clinical Trials Registry (ChiCTR 1800016991). Figure 1 shows the research design. 


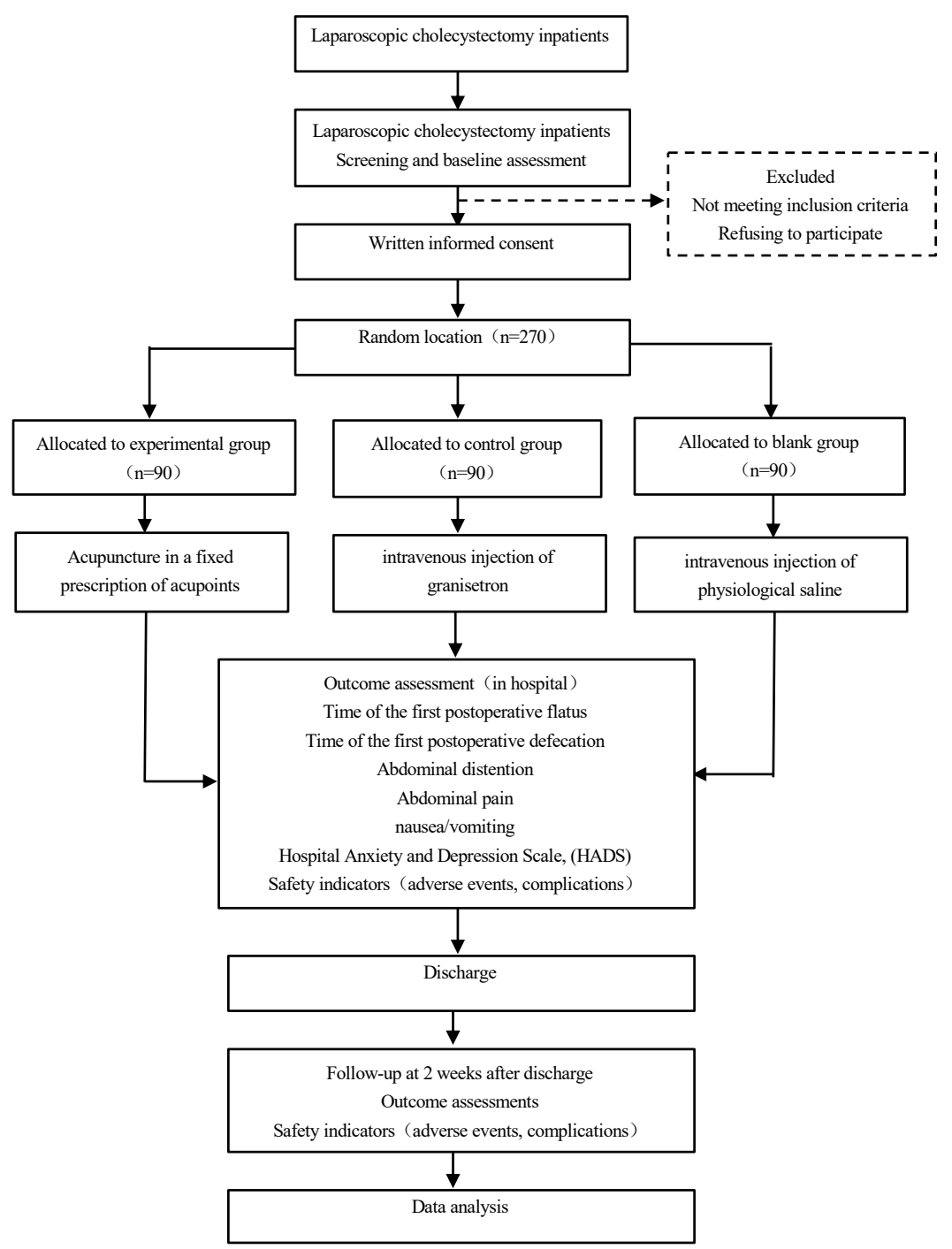

Fig. 1 Trial flow chart.

\section{Participants}

All subjects will be diagnosed by 2 surgical experts and 1 anesthesiologist according to the inclusion and exclusion criteria. The potential participants who fulfill all the inclusion criteria and match none of the exclusion criteria will be required to have face-to-face discussion with the research assistant about the study. The research assistant will conduct a series of disease assessment and safety assessment to eligible patients who are interested in participating. Subjects will be randomly divided into three groups and received different treatment after they sign the informed consent.

\section{Recruitment}

During the study, three research assistants will go to the general surgery department of the research centers three times a week to screen subjects, eligible patients will be identified and invited to participate in the study. The research assistants will give written informed consent to the patient and explain the details of the study such as the objectives, scope, procedure, potential benefits, and risks. The patient will sign the informed consent in the presence of the research assistants, who can also accept oral informed consent if the participants are unable to read. 


\section{Sample Size Calculation}

The total sample size of the study is calculated according to the formula of total effective rate of two groups: $\mathrm{n}=2\left(U_{\alpha}+U_{\beta}\right)^{2} P(1-P) /\left(P_{1}-P_{2}\right)^{2}$. It is known that the total effective rate of acupuncture for postoperative gastrointestinal dysfunction is $84.8 \%$, which is better than that of granisetron (69.4\%). $P_{1}$ is the effective rate of the experimental group, $P_{2}$ is the effective rate of the control group, $P=P_{1}+P_{2} / 2$, assuming $\alpha=0.05, \beta=0.1$, the calculated result is $\mathrm{n} \approx 153$. If the dropout rate is $15 \%, 180$ subjects ( 90 in each group) are needed in the two groups, so 270 subjects are needed in the three groups.

\section{Inclusion criteria}

Subjects will be included if they accord with all the following criteria of the study: (1) the age is between 18-70 years old; (2) the operation type is laparoscopy, and the anesthesia type is general anesthesia, which meets the I-II classification standard of American Society of anesthesiologists (ASA); (3) the intravenous analgesia pump is used postoperatively; (4) the operation time is $2 \pm$ $1.0 \mathrm{~h}$; (5) the weight is $45-80 \mathrm{~kg}, 18.5<\mathrm{BMI}<26\left(\mathrm{~kg} / \mathrm{m}^{2}\right)$; (6) have no any other clinical trials during this study; (7) have no cognitive impairment, aphasia, mental disorder, and other communication barriers; (8) participate in the trial and sign the informed consent voluntarily.

\section{Exclusion criteria}

Subjects will be excluded if they fit any of the following criteria: (1) obesity, pregnancy, lactation, have a fertility or pregnancy plan within recent 3 months; (2) with severe cardiovascular disease, central nervous system disease, diabetes mellitus, and psychosis; (3) with gastric motility drugs using within 24 hours before operation or gastric tube keeping after operation; (4) with severe gastrointestinal disease or previous history of postoperative gastrointestinal dysfunction; (5) with other type of anesthesia, or ASA grade $\geq \mathrm{III}$, operation time $>180 \mathrm{~min}$, or undergo other operations besides laparoscopy; (6) with aversion to acupuncture treatment, or have aphasia, audio-visual disorders; (7) they participated in other clinical trials within one month; (8) with intraoperative blood loss $\geq 1000 \mathrm{ml}$ or hemoglobin $<70 \mathrm{~g} / \mathrm{L} ;(9)$ with the history of drug abuse.

\section{Elimination standard}

Subjects will be eliminated from the trial if they fit any of the following conditions: (1) laparoscopy must be converted to laparotomy;(2) they refuse to continue treatment for various reasons; 3 ) they have severe syndromes such as biliary fistula and peritonitis during the treatment; 4) they are transferred to other specialist treatment;(5) they became comatose or die;(6) they cant not participate in the follow-up for various reasons;(7) researchers accidentally included the subjects who do not meet the inclusion criteria;(8) they have less compliance with the treatment regulations and failed to provide information that might be important for the evaluation.

\section{Randomization, allocation concealment}

SPSS 25.0 will be used to generate random number, A specially appointed researcher is responsible for random grouping to reduce selection bias and confounding factors. Subjects will be randomly assigned to the experimental group, the control group and the blank group in the proportion of 1:1:1, and each group will contain 90 participants. The recruiters will obtain the sequence number from the assigning researcher when eligible subjects participate in the trial. To further reduce the bias and prevent communication, Subjects from the three groups will be treated in separate rooms. 
To prevent researchers' prejudice from confusing the results, this study will use sealed envelope method to blind randomization. The distribution sequence number of subjects will be placed in the sealed and opaque envelope, so that the recruiters and evaluators will not know the allocations. To ensure strict confidentiality, the envelope is not transparent even under strong light. The subject's name and birthdates will be written on the outside of the envelope using carbon paper to avoid confusion. The information on the sealed envelope and the subject's details will be recorded using a video, and a copy of the outer label of the sealed letter will be attached to the group distribution card in the envelope. Then the video will be checked by another researcher to make sure the envelopes are sealed. The whole process of opening the envelopes will also be recorded by the video.

\section{Blinding}

Due to the characteristics of acupuncture clinical trials and the high popularity of acupuncture in China, it is impossible to completely blind subjects in this trial, nor to make acupuncturists blind in treatment allocation. As a result, acupuncturists will not participate in the assessment process, and different groups will receive treatment in different rooms to guarantee it impossible for participants to influence the group allocation. The evaluators and statisticians will work independently and be unaware of the group allocation throughout the whole study.

\section{Intervention}

The treatment strategy of this study is collectively formulated by experienced acupuncturists, surgeons and anesthesiologists. After laparoscopic surgery, all the subjects will fasting and in supine position without pillow, the intervention will be conducted30 minutes after surgery. The experimental group will be treated with sterile disposable stainless steel needles (Huatuo medical instruments Co. Ltd., Suzhou, China; $0.3 \mathrm{~mm} \times 40 \mathrm{~mm} / 0.3 \mathrm{~mm} \times 25 \mathrm{~mm}$ ). Acupoints will be located in accordance with the World Health Organization Standard Acupuncture Point Locations in the Western Pacific Region ${ }^{[28]}$. The fixed prescription includes Hegu (LI4), Neiguan (PC6), Zusanli (ST36), Gongsun (SP4), lateral line 2 of forehead (MS3), lateral line 3 of forehead (MS4), and middle line of vertex (MS5)(shown as Fig.2). The acupuncture needles will insert into acupoints after the routine sterilization. After reaching the standard depth, the acupuncture needles will be evenly lifted, inserted and twisted to de qi, the operation of each acupoint will last for 30 seconds. the needles will be kept on the trunk and limbs for 30 minutes and on the head for 6 hours after $d e$ $q i$. All acupuncture treatments were performed by two acupuncturists registered in China and with more than 3 years of clinical experience. The acupuncturist will be trained before the experiment, The training courses include acupoint positioning, acupuncture operation skills, and communication skills. The control group will be treated with intravenous injection of $3 \mathrm{mg} / 3 \mathrm{ml}$ granisetron (drug approval No. H20030161, Sichuan Taiji Pharmaceutical Co., Ltd). Blank group will be treated with intravenous injection of $3 \mathrm{ml}$ physiological saline (drug approval No. H33021575, Hangzhou Minsheng Pharmaceutical Co., Ltd) 

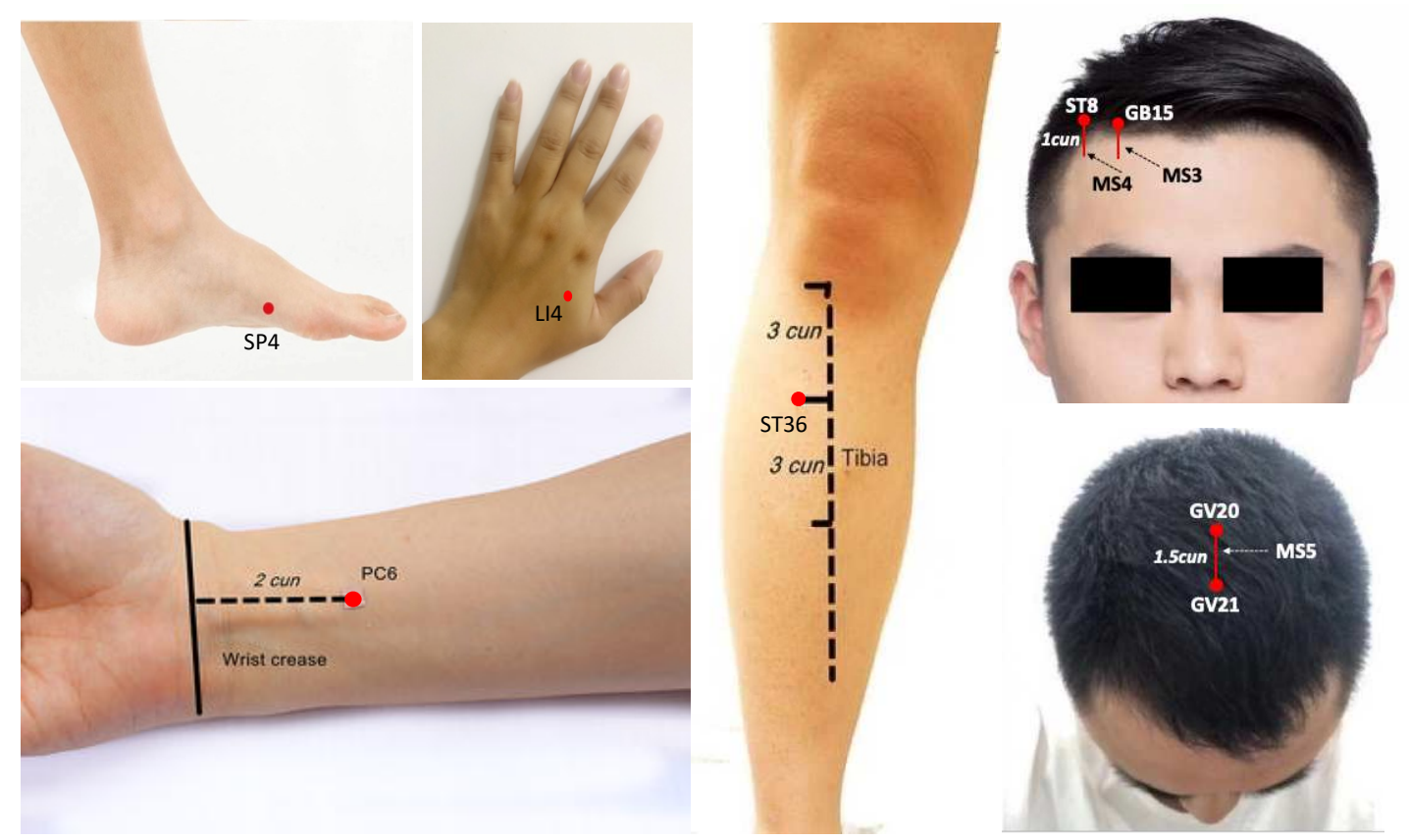

Fig.2 The acupoints location of the fixed acupuncture prescription

\section{Outcome indicators}

\section{Baseline data}

The following baseline data will be recorded using the established personal information questionnaire: demographic characteristics such as gender, age, height, and weight; general clinical conditions such as operation time, bleeding volume, anesthesia time, and intraoperative infusion volume; basic vital signs such as heart rate, blood oxygen, blood pressure, and respiration.

\section{Primary outcome}

Time of the first postoperative flatus: the researcher will inform the subjects and their families to assist in recording time of the first postoperative flatus, which is the interval between the time of subjects returning to the ward after the operation to the time of first flatus after operation.

\section{Secondary outcome}

(1) Time of the first postoperative defecation: the researcher will inform the subjects and their families to assist in recording time of the first postoperative defecation, which is the interval between the time of subjects returning to the ward after the operation to the time of first defecation after operation.

(2) Abdominal pain: the Visual Analog Scale(VAS, Fig.3)will be used to assess the degree of postoperative abdominal pain of subjects ${ }^{[29]}$, which will be recorded by researchers at $\mathrm{t}_{1}-\mathrm{t}_{5}$.

Abdominal distention: Likert scale(see Table 1)will be used to evaluate the degree of abdominal distention ${ }^{[30]}$, which will be recorded by researchers at $\mathrm{t}_{1}-\mathrm{t}_{5}$.

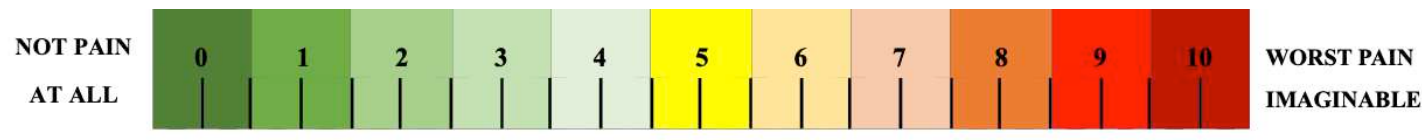

Fig. 3 Visual analog scale 
Table 1 Presentation of the Likert-type scale

\begin{tabular}{ll}
\hline Score & Explanation \\
\hline 0 & I feel no abdominal distension at all \\
1 & I feel a little of abdominal distension \\
2 & I feel abdominal distension but I can bear \\
3 & I feel obvious abdominal distension that I cannot bear but it doesn't affect my life \\
4 & I feel horrible abdominal distension and need it addressed \\
\hline
\end{tabular}

(3)Nausea: the VAS scale will be used to assess the degree of nausea ${ }^{[29]}$. The subjects and their families will assist in recording the degree of nausea at $\mathrm{t}_{1}-\mathrm{t}_{5}$.

(4) Vomiting: The subjects and their families will assist in recording the times of vomiting at $\mathrm{t}_{1}-\mathrm{t}_{5}$.

(5)Gastrointestinal hormones: blood samples will be taken 8 hours before operation, 30 minutes after operation, and 24 hours after operation to detect the level of gastrointestinal hormones. We will use Enzyme-linked immunosorbent assay (ELISA) to detect the levels of serum gastrin, motilin, ghrelin, serotonin in the three groups of subjects. In the fasting state, $3 \mathrm{ml}$ blood will be collected from the vein. The blood samples will be kept at room temperature for 30 minutes, then centrifuged at $3500 \mathrm{rpm}$ for 10 minutes, and the serum will be stored in a refrigerator at $-70^{\circ} \mathrm{C}$ for testing. Serum samples will be collected by clinical centers and relevant tests will be conducted by Chengdu Lilai Biotechnology Co., Ltd.

(6) Psychological status: The Hospital Anxiety and Depression Scale (HADS) will be used to evaluate the psychological status of the subjects ${ }^{[31]}$. HADS has two subscales of anxiety and depression. There are seven problems for anxiety and depression respectively. The anxiety and depression can be indicated by the score of 0-7 points (no symptoms), 8-10 points (suspicious symptoms), 11-21 points (obvious symptoms). The first evaluation will be conducted 8 hours before the operation and the second evaluation will be conducted on the day of discharge.

\section{Outcome measurements}

The researchers will make a decision on whether to include the subjects in the trial 1 day before the laparoscopic surgery, and the first evaluation will be conducted on this 0 day of the trial. Subjects will receive an intervention 30 minutes after surgery. The SPIRIT figure of the subject recruitment, intervention and evaluation schedule is shown in Figure 4.

\section{Adverse events and complications}

The participants of this study should voluntarily report information about adverse events to researchers, such as pallor, fainting during acupuncture treatment, sweating, dizziness, local hematoma, bleeding, unbearable prickling, and continuous severe pain more than $1 \mathrm{~h}$ after acupuncture. The adverse events related to the acupuncture such as sticking of the needle, broken needles, and bent needles will be reported by acupuncturists. All adverse events must be fully registered on the adverse events page of case report forms (CRFs). Researchers will identify the occurrence of adverse events and record all details such as the date, time, extent, and correlation with treatment. Data on postoperative complications will be recorded during hospitalization and within 2 weeks after discharge, any complications that threaten the life of the subjects or serious adverse events (SAE) will also be recorded. The researchers will seek emergency medical assistance as well as report the SAE immediately. 


\begin{tabular}{|c|c|c|c|c|c|c|c|c|c|}
\hline \multirow[b]{3}{*}{ TIMEPOINT } & \multicolumn{9}{|c|}{ STUDY PERIOD } \\
\hline & \multirow{2}{*}{$\begin{array}{c}\text { Enrolment } \\
-t_{1}\end{array}$} & \multirow{2}{*}{$\begin{array}{c}\text { Allocation } \\
t_{0}\end{array}$} & \multicolumn{5}{|c|}{ Post-allocation } & \multicolumn{2}{|c|}{ Close-out } \\
\hline & & & $\mathrm{t}_{1}$ & $\mathrm{t}_{2}$ & $t_{3}$ & $\mathrm{t}_{4}$ & $\mathrm{t}_{5}$ & $\mathrm{t}_{6}$ & $\mathrm{t}_{7}$ \\
\hline \multicolumn{10}{|l|}{ ENROLMENT: } \\
\hline Eligibility screen & $x$ & & & & & & & & \\
\hline Informed consent & $x$ & & & & & & & & \\
\hline Allocation & & $x$ & & & & & & & \\
\hline \multicolumn{10}{|l|}{ INTERVENTIONS: } \\
\hline Experimental group & & & $x$ & & & & & & \\
\hline control group & & & $x$ & & & & & & \\
\hline Blank group & & & $x$ & & & & & & \\
\hline \multicolumn{10}{|l|}{ ASSESSMENTS: } \\
\hline Baseline variables & $x$ & $x$ & & & & & & & \\
\hline Time of the first postoperative flatus & & & $x$ & $x$ & $x$ & $x$ & $x$ & & \\
\hline Time of the first postoperative defecation & & & $x$ & $x$ & $x$ & $x$ & $x$ & & \\
\hline Time of the first postoperative defecation & & & $x$ & $x$ & $x$ & $x$ & $x$ & & \\
\hline Abdominal distension & & & $x$ & $x$ & $x$ & $x$ & $x$ & & \\
\hline Blood gastrointestinal hormones & & $x$ & $x$ & & & $x$ & & & \\
\hline Nausea & & & $x$ & $x$ & $x$ & $x$ & $x$ & & \\
\hline Psychological status & & $x$ & & & & & & $x$ & \\
\hline Adverse events & & & $x$ & $x$ & $x$ & $x$ & $x$ & $x$ & $x$ \\
\hline Postoperative complications & & & $x$ & $x$ & $x$ & $x$ & $x$ & $x$ & $x$ \\
\hline Compliance evaluation & & & $x$ & $x$ & $x$ & $x$ & $x$ & $x$ & $x$ \\
\hline
\end{tabular}

Fig. 4 SPIRIT Figure for acupuncture for gastrointestinal dysfunction after laparoscopy

The schedule of enrollment, interventions, and assessments. $-t_{1}$ is the day before the surgery, $t_{0}$ is the day of the surgery, $t_{1}$ is $0-2$ hours after the surgery, $t_{2}$ is $2-6$ hours after the surgery, $t_{3}$ is $6-12$ hours after the surgery, $t_{4}$ is $12-24$ hours after the surgery, $t_{5}$ is $24-48$ hours after the surgery, $t_{6}$ is the day of the discharged, $\mathrm{t}_{7}$ is 2 weeks after patients discharged from the hospital.

\section{Data collecting and monitoring}


The result data of this study will be recorded on the paper CRFs by a special evaluator. In order to facilitate the observation of indicators and data collection, the CRF will contain all information such as observation time, observation indicators, adverse event records, and safety assessment. The result evaluator is required to fill in the relevant information quickly and accurately according to the principle of CRF. The evidence-based medicine center of Chengdu University of traditional Chinese medicine will be responsible for monitoring the data every three months. The acupuncturists and statisticians can not access or obtain the data during the whole evaluation process.

\section{Quality control}

To ensure the quality of the study, methodology experts, acupuncturists, surgeons, and anesthesiologists have discussed and revised the protocol for many times. Before the start of the project, all researchers must receive unified training that including research scheme, test process, operation specification, etc. The quality controller directly appointed by the project leader will check the CRF every two weeks. The original data on the CRF cannot be modified. If it needs to be modified, the reason for the modification shall be stated and the name of the modifier shall be written next to the modification. The conference will be hold by researchers every three months to report and discuss the process of the study.

\section{Statistical analysis}

An independent statistician will analysis the outcome data, which will be analyzed in accordance with the principle of intention-to-treat (ITT), i.e. all subjects participate in the group will be included. The analysis of efficacy and safety indicators will be conducted in accordance with the principle of per-protocol (PP), i.e. all subjects who completed the whole trial process without major protocol violations will be included. All statistical analysis will be based on ITT population and the results of ITT analysis will be compared with PP analysis to assess the sensitivity. All missing data will be analyzed with the last observation carried forward (LOCF) interpolation method. The qualitative data will be tested for normality, according to which independent t-test or Mann Whitney U-test were used for group comparison. The continuous variables will be represented by the statistical description of $\bar{\chi} \pm$ SD of the normal distribution data. The categorical variables will be represented by numbers (percentages) using $\chi^{2}$ test or Fisher exact test $P<0.05$ will be considered statistically significant on both sides in all the statistical tests and SPSS 25.0 software (SPSS, SPSS Inc., Chicago, USA) will be used for data statistical analysis.

\section{Discussion}

The main factors affecting the postoperative rehabilitation of patients are gastrointestinal dysfunction, pain, and inconvenience occurred by operation ${ }^{[32]}$. The application of opioid analgesics can also improve the incidence of postoperative gastrointestinal dysfunction, in 2009, the incidence of postoperative gastrointestinal dysfunction caused by opioid analgesics in the United States was as high as $81 \%{ }^{[33]}$. The prevalence of postoperative gastrointestinal dysfunction in China is difficult to estimate due to the lack of reliable Evidence-Based Medicine reports. However, according to clinical observation ${ }^{[34]}$, most patients are upset by a series of obvious discomfort syndrome caused by postoperative gastrointestinal dysfunction, which seriously affects the prognosis of patients. However, the usual treatment measures are far from meeting the requirements of patients, so clinicians and researchers must find some better measures to promote the early recovery of 
gastrointestinal function. The ideal postoperative rehabilitation measures can not only effectively in promoting the gastrointestinal function but also with minimal side effects.

In China, acupuncture is one of alternative therapies that inserts the fine stainless steel needle into specific parts of the body, which is widely used to treat various diseases. Acupuncture is an important part of traditional Chinese medicine, which has been used in China for more than 3000 years. Deqi is the basis of curative effect no matter stimulating acupoints by hand acupuncture or electroacupuncture. Deqi refers to a special feeling brought by acupuncture, such as acid, numbness, heaviness, distention, etc. Most acupoints have clear international general positioning and codes so that the technology of acupuncture is easy to master and train that. However, because of the different philosophical basis between TCM and translational medicine, it is difficult to bring acupuncture into the mainstream treatment program. Fortunately, the emergence of molecular biology, neuroendocrinology, and immunology methods enable researchers to apply modern technology to acupuncture research and reveal the mechanism of acupuncture.

Many studies have shown that acupuncture can promote the recovery of postoperative gastrointestinal dysfunction but few of them are high-quality studies. In order to provide EvidenceBased medicine proof, and verify the efficacy and safety of acupuncture in the treatment of postoperative gastrointestinal dysfunction, we designed this clinical study. Based on the theoretical research of acupuncture and long-term clinical experience, we hypothesize that acupuncture may promote the recovery of gastrointestinal dysfunction after laparoscopic surgery by regulating the level of gastrointestinal hormones. A systematic review ${ }^{[35]}$ indicated that the time of first flatus after operation is of great clinical significance in the recovery of gastrointestinal motility. Most clinical trials of postoperative gastrointestinal dysfunction are treating the time of first postoperative flatus as the primary result, so we will also use it as the main outcome index. Postoperative gastrointestinal dysfunction can cause emotional impact on patients ${ }^{[36]}$ so that the anxiety and depression of the subjects will also act as the secondary observation indexes. In the inclusion criteria of this study, subjects aged 18-70 will be selected to cover as wide a range as possible. At the same time, exclusion of participants taking gastrointestinal motility related drugs is essential for accurate clinical results. During the study, patients will be allowed to use rescue drugs when the abdominal pain, abdominal distension, nausea, vomiting and other symptoms can not be beard, this strategy reflects the clinical practice in the real world and meets the moral obligation.

This study has some limitations. In this study, we design the drug and blank control group, but not design the placebo sham acupuncture control group. In the previous experiment, we found that even if intradermal acupuncture or transcutaneous electrical stimulation could lead to physiological effects, which may be caused by the specificity of acupoints. At present, There is a lack of internationally recognized criterion and mature method of sham acupuncture ${ }^{[37]}$. In addition, it is difficult to blind subjects because most of Chinese patients have the experience of acupuncture, which makes the trial lack of sham acupuncture control group. In summary, this study protocol describes a randomized, evaluated blinded, multi-center clinical controlled trial. The results of this study will provide information on the effectiveness, safety, and feasibility of acupuncture for gastrointestinal dysfunction after laparoscopic surgery, the purpose of this study is to provide reliable reference for promoting the clinical application of this therapy.

\section{Trial status}


The research started on December 18, 2018 and is now in progress. So far, 200 participants have been recruited and 180 participants have completed the intervention. The admission of all subjects is expected to be completed by March 31, 2020.

\begin{abstract}
Abbreviations
CRF: Case Report Form; TCM: Traditional Chinese medicine; HADS: Hospital Anxiety and Depression Scale; VAS: Visual analog scale; CRF: Case report form; ITT: Intention-to-treat; PP: Per-protocol;PC6: Neiguan; ST36: Zusanli; LI4:Hegu;SP4: Gongsun; CONSORT: Consolidated Standards of Reporting Trials; GCP: Good Clinical Practice; ITT: Intention-to-treat; LOCF: Last observation carried forward; SPIRIT: Standard Protocol Items Recommendations for Interventional Trials; SPSS: Statistical Package for the Social Sciences; ELISA: Enzyme-Linked Immunosorbent Assay; RCT: Randomized Controlled Trial; AE: Adverse event.
\end{abstract}

\title{
Declarations
}

\section{Ethics approval and consent to participate}

This study has been approved by Sichuan Regional Ethics Review Committee of TCM (Approval No.18KL-044) and ethical approval applies to all clinical research centers. The clinical trial has been registered in China Clinical Trial Registry, ChiCTR 1800016991 and its implementation follows the principles of the Declaration of Helsinki (Edinburgh 2000). The study will only include those who signed the written informed consent.

\section{Consent for publication}

Two subjects' recognizable images were included in this study plan, and we have obtained their written informed consent for publishing the image data.

\section{Availability of data and materials}

The datasets used and analysed during the current study are available from the first author on reasonable request.

\section{Competing interests}

The authors declare that they have no competing interests.

\section{Funding}

The funding of this study comes from the Key Research and Development Plan of Sichuan Provincial Department of Science and Technology - "Research on the effect of acupuncture on hormone level of patients with gastrointestinal dysfunction after general anesthesia" (No: 18ZDFY0347). Funders have no role in research design, data collection, management, analysis or interpretation, report writing or submission decisions; they have no final authority over any of these activities.

\section{Authors' contributions}

Conceptualization: Lisha Liu, Xiuli Yuan, Lei Yang.

Data curation: Lisha Liu, Xiuli Yuan.

Formal analysis: Lisha Liu, Xiuli Yuan. 
Funding acquisition: Lisha Liu, Lei Yang.

Investigation: Lisha Liu, Xiuli Yuan, QuanminJiang,Lei Yang, Zhang Jingyuan.

Methodology: Lisha Liu, Xiuli Yuan, Lei Yang.

Project administration: Lisha Liu, Xiuli Yuan.

Supervision: Lisha Liu, Xiuli Yuan, Lei Yang.

Validation: Lisha Liu, Xiuli Yuan, Peng Xuanfu.

Writing - original draft: Lisha Liu, Xiuli Yuan, QuanminJiang, Lei Yang.

Writing - review \& editing: Lisha Liu, Xiuli Yuan, Jian Huo, Luo Jing.

\section{Acknowledgements}

Not applicable.

\section{Authors' information}

Lisha Liu, Email:+86 18708174822, wenliyinyusha@163.com, orcid: 0000-0001-8965-4366

Xiuli Yuan, Email:+86 13881109129, 573859636@qq.com

Quanmin Jiang, Email:1142952862@qq.com

Lei Yang, Email:5280263@qq.com

Guangqiang Huang, Email:1690673167@qq.com

Jingyuan Zhang, Email:571703993@qq.com

Jing Luo, Email:3122063669@qq.com

Xiaoying Liu, Email:10126056@qq.com

Jian Huo, Email:577311381@qq.com

\section{References}

[1] Saito S, Nakamura M, Hosoya Y, et al. Postoperative quality of life and dysfunction in patients after combined total gastrectomy and esophagectomy. Ann Med Surg (Lond). 2017;22:34-38.

[2] Xue DD, Cheng $\mathrm{Y}, \mathrm{Wu} \mathrm{M}$, et al. Comprehensive geriatric assessment prediction of postoperative complications in gastrointestinal cancer patients: a meta-analysis. Clin Interv Aging. 2018;13:723-736.

[3] Ge W, Chen G, Ding YT. Effect of chewing gum on the postoperative recovery of gastrointestinal function. Int J Clin Exp Med. 2015;8(8):11936-11942.

[4] Wanjura V, Lundström $P$, Osterberg $J$, et al. Gastrointestinal quality-of-life after cholecystectomy: indication predicts gastrointestinal symptoms and abdominal pain. World $\mathrm{J}$ Surg. 2014;38(12):3075-3081.

[5] Carlisle J, Stevenson CA. WITHDRAWN: Drugs for preventing postoperative nausea and vomiting. Cochrane Database Syst Rev. 2017;7(7):CD004125.

[6] Feldheiser A, Aziz O, Baldini G, et al. Enhanced Recovery After Surgery (ERAS) for gastrointestinal surgery, part 2: consensus statement for anaesthesia practice. Acta Anaesthesiol Scand. 2016;60(3):289-334.

[7] Takahashi T. Mechanism of acupuncture on neuromodulation in the gut--a review. Neuromodulation 2011; 14: 8-12.

[8] $\mathrm{Li} \mathrm{H}, \mathrm{He} \mathrm{T}, \mathrm{Xu} \mathrm{Q}$, et al. Acupuncture and regulation of gastrointestinal function. World $\mathrm{J}$ Gastroenterol. 2015;21(27):8304-8313. 
[9] Zhang F, Wu L, Zhao J, et al. Neurobiological Mechanism of Acupuncture for Relieving Visceral Pain of Gastrointestinal Origin. Gastroenterol Res Pract. 2017;2017:5687496.

[10] Lu MJ, Yu Z, He Y, et al. Electroacupuncture at ST36 modulates gastric motility via vagovagal and sympathetic reflexes in rats. World J Gastroenterol. 2019;25(19):2315-2326.

[11] Zhang Z, Wang C, Li Q, et al. Electroacupuncture at ST36 accelerates the recovery of gastrointestinal motility after colorectal surgery: a randomised controlled trial. Acupunct Med. 2014;32(3):223-226.

[12] Tjen-A-Looi SC, Guo Z-L, Longhurst JC. GABA in nucleus tractus solitarius participates in electroacupuncture modulation of cardiopulmonary bradycardia reflex. Am J Physiol Regul Integr Comp Physiol. 2014;307(11):R1313-1323.

[13] Khlevner J, Park Y, Margolis KG. Brain-Gut Axis: Clinical Implications. Gastroenterol Clin North Am. 2018;47(4):727-739.

[14] Tsang SW, Auyeung KK, Bian ZX, et al. Pathogenesis, Experimental Models and Contemporary Pharmacotherapy of Irritable Bowel Syndrome: Story About the Brain-Gut Axis. Curr Neuropharmacol. 2016;14(8):842-856.

[15] Sikiric P. EDITORIAL (Thematic Issue: Brain Gut Axis-New View). Curr Neuropharmacol. 2016;14(8):840-841.

[16] Sanger GJ, Furness JB. Ghrelin and motilin receptors as drug targets for gastrointestinal disorders. Nat Rev Gastroenterol Hepatol. 2016;13(1):38-48.

[17] Kamiji MM, Troncon LE, Antunes-Rodrigues J, Elias LL, de Castro M, Oliveira RB. Ghrelin and PYY (3-36) in gastrectomized and vagotomized patients: relations with appetite, energy intake and resting energy expenditure. Eur J Clin Nutr. 2010;64(8):845-852.

[18] Sun J, Wu X, Meng Y, et al. Electro-acupuncture decreases 5-HT, CGRP and increases NPY in the brain-gut axis in two rat models of Diarrhea-predominant irritable bowel syndrome(DIBS). BMC Complement Altern Med. 2015;15:340.

[19] Morey SS. NIH issues consensus statement on acupuncture. Am Fam Physician. 1998;57(10):2545-2456.

[20] Wang Shiyuan, Gu Muen ,Jin Zhu,et al. Meta-analysis of Simple Acupuncture to Promote Postoperative Abdominal Gastrointestinal Dysfunction Recovery. World Chin Med, 2018;13(11),2911-2920.

[21] Han Xu. A review on treating postoperative gastrointestinal disorders by acupuncture. Clinical J of Chin Med.2018;10(11), 138-141.

[22] Li Jinjin, Shao Xiaomei, Zhao Wensheng, et al. A Systematic Review and Meta-analysis to Acupuncture on Postoperative Ileus .2015;39(02), 162-166.

[23] Chung WY, Liu SY, Gao JC, et al. Modulatory effect of International Standard Scalp Acupuncture on brain activation in the elderly as revealed by resting-state fMRI. Neural Regen Res. 2019;14(12):2126-2131.

[24] ZHENG, Chun-li, WANG Jian, et al. Acupoint Rules of Acupuncture and Moxibustion for the Treatment of Gastrointestinal Dysfunction. Journal of Clinical Acupuncture and Moxibustion. 2015;31(09), 52-55.

[25] Moher D, Schula KF, Altman DG. CONSORT statement: revised recommendations for improving the quality of reports of parallel group randomized trials. BMC Med Res Methodol. $2001 ; 1: 2$. 
[26] MacPherson H, White A, Cummings $M$, et al. Standards for reporting interventions in controlled trials of acupuncture: the STRICTA recommendations. Complement Ther Med. 2001;9(4):246-249.

[27] Chan AW, Tetzlaff JM, Altman DG, et al. SPIRIT 2013 statement: defining standard protocol items for clinical trials. Ann Intern Med. 2013;158:200-207.

[28] Organization W. WHO standard acupuncture point locations in the Western Pacific Region. World Health Organization, 2008.

[29] Tan Y, Zhao Y, He T, et al. Efficacy and safety of auricular point acupressure treatment for gastrointestinal dysfunction after laparoscopic cholecystectomy: study protocol for a randomized controlled trial. Trials. 2016;17(1):280.

[30] Liu MY, Wang CW, Wu ZP, et al. Electroacupuncture for the prevention of postoperative gastrointestinal dysfunction in patients undergoing vascular surgery under general anesthesia: study protocol for a prospective practical randomized controlled trial. J Integr Med. 2014;12(6):512-519.

[31] Bjelland I, Dahl AA, Haug TT, et al. The validity of the Hospital Anxiety and Depression Scale. An updated literature review. J Psychosom Res. 2002;52(2):69-77.

[32] Gan TJ. Poorly controlled postoperative pain: prevalence, consequences, and prevention. J Pain Res. 2017;10:2287-2298.

[33] Bell T. J, Panchal S. J, Miaskowski C, et al, Williamson R. The prevalence, severity, and impact of opioid-induced bowel dysfunction: results of a US and European patient survey (PROBE 1) Pain Medicine. 2009;10(1):35-42.

[34] McCall MD, Graham PJ, Bathe OF. Quality of life: A critical outcome for all surgical treatments of gastric cancer. World J Gastroenterol. 2016;22(3): 1101-1113.

[35] Wallström A, Frisman GH. Facilitating early recovery of bowel motility after colorectal surgery: a systematic review. J Clin Nurs. 2014;23(1-2):24-44.

[36] Ghoneim MM, O'Hara MW. Depression and postoperative complications: an overview. BMC Surg. 2016;16:5.

[37] Zhang CS, Tan HY, Zhang GS, et al. Placebo Devices as Effective Control Methods in Acupuncture Clinical Trials: A Systematic Review. PLoS One. 2015;10(11):e0140825. 


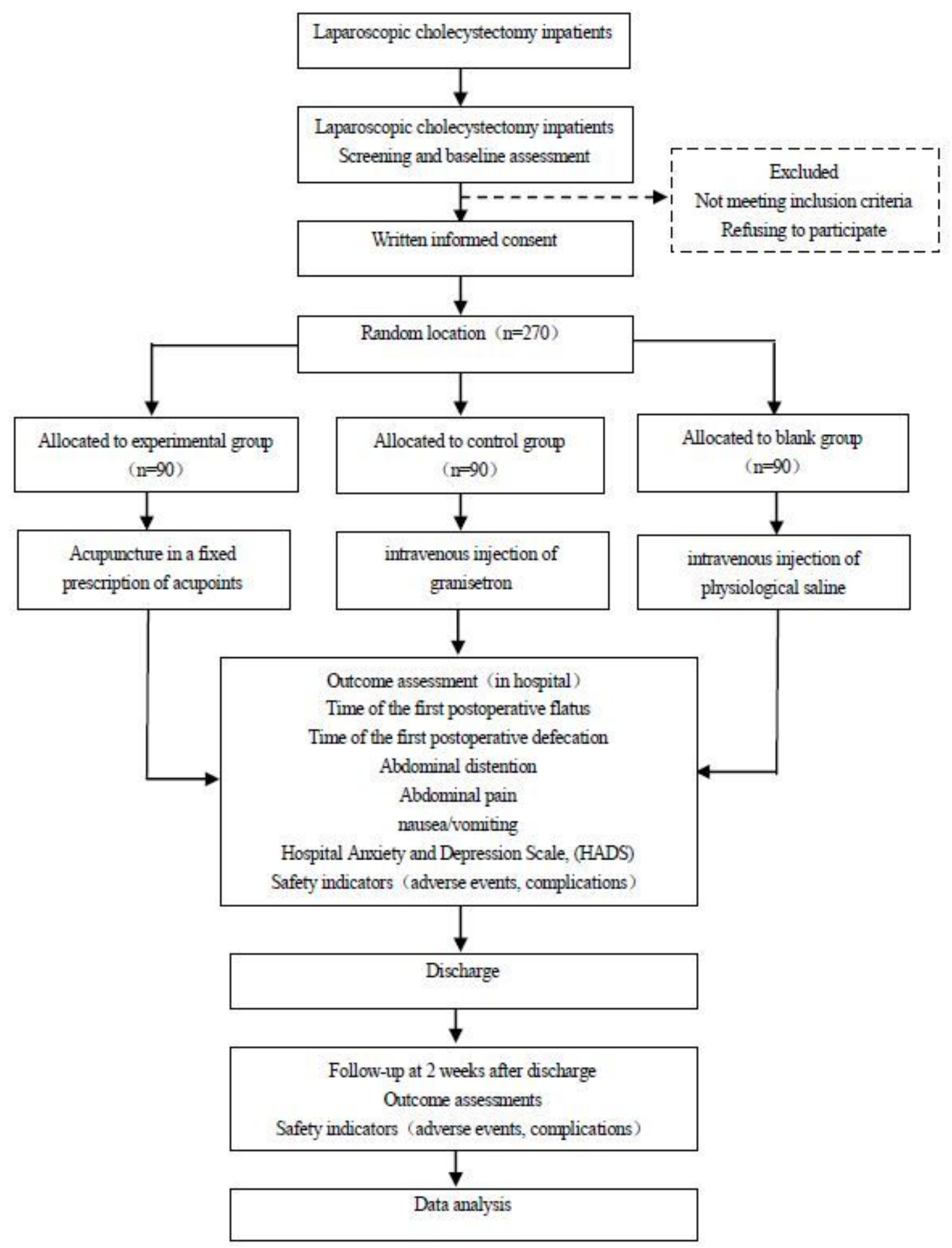

\section{Figure 1}

Trial flow chart. 

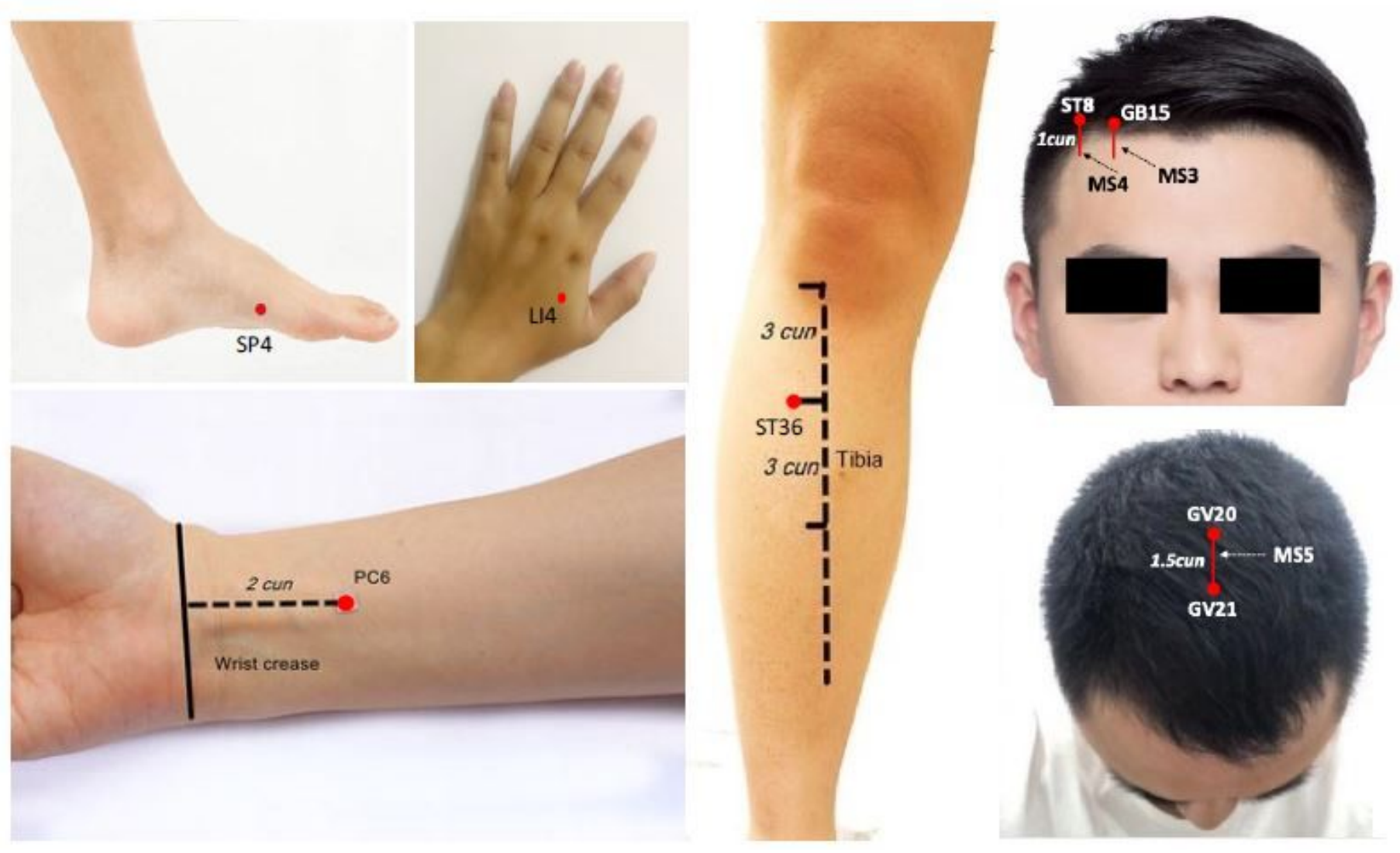

Figure 2

The acupoints location of the fixed acupuncture prescription

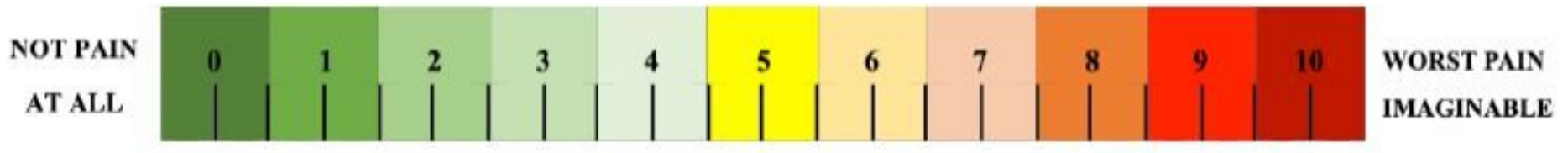

Figure 3

Visual analog scale 


\begin{tabular}{|c|c|c|c|c|c|c|c|c|c|}
\hline & \multicolumn{9}{|c|}{ STUDY PERIOD } \\
\hline \multirow[b]{2}{*}{ TIMEPOINT } & Enrolment & Allocation & \multicolumn{5}{|c|}{ Post-allocation } & \multicolumn{2}{|c|}{ Close-out } \\
\hline & $-t_{1}$ & $\mathrm{t}_{0}$ & $\mathrm{t}_{1}$ & $\mathrm{t}_{2}$ & $\mathrm{t}_{3}$ & $\mathrm{t}_{4}$ & $t_{5}$ & $t_{6}$ & $t_{7}$ \\
\hline \multicolumn{10}{|l|}{ ENROLMENT: } \\
\hline Eligibility screen & $x$ & & & & & & & & \\
\hline Informed consent & $x$ & & & & & & & & \\
\hline Allocation & & $x$ & & & & & & & \\
\hline \multicolumn{10}{|l|}{ INTERVENTIONS: } \\
\hline Experimental group & & & $x$ & & & & & & \\
\hline control group & & & $x$ & & & & & & \\
\hline Blank group & & & $x$ & & & & & & \\
\hline \multicolumn{10}{|l|}{ ASSESSMENTS: } \\
\hline Baseline variables & $x$ & $x$ & & & & & & & \\
\hline Time of the first postoperative flatus & & & $x$ & $x$ & $\times$ & $x$ & $\times$ & & \\
\hline Time of the first postoperative defecation & & & $x$ & $x$ & $x$ & $x$ & $\times$ & & \\
\hline Time of the first postoperative defecation & & & $x$ & $x$ & $x$ & $x$ & $\times$ & & \\
\hline Abdominal distension & & & $x$ & $x$ & $x$ & $x$ & $x$ & & \\
\hline Blood gastrointestinal hormones & & $x$ & $x$ & & & $x$ & & & \\
\hline Nausea & & & $x$ & $x$ & $\times$ & $x$ & $\times$ & & \\
\hline Psychological status & & $x$ & & & & & & $\times$ & \\
\hline Adverse events & & & $x$ & $x$ & $x$ & $x$ & $\times$ & $x$ & $x$ \\
\hline Postoperative complications & & & $x$ & $x$ & $x$ & $x$ & $\times$ & $\times$ & $x$ \\
\hline Compliance evaluation & & & $x$ & $x$ & $x$ & $x$ & $\times$ & $\times$ & $x$ \\
\hline
\end{tabular}

Figure 4

SPIRIT figure for acupuncture for gastrointestinal dysfunction after laparoscopy

\section{Supplementary Files}

This is a list of supplementary files associated with this preprint. Click to download. 
- SPIRIT2013checklistandfigure.docx 